\title{
Macrophage inflammatory factors promote epithelial- mesenchymal transition in breast cancer
}

\author{
Robert B. Bednarczyk ${ }^{1}$, Neha Y. Tuli ${ }^{1}$, Elyse K. Hanly ${ }^{1}$, Ghada Ben Rahoma ${ }^{1}$, \\ Rachana Maniyar ${ }^{1}$, Abraham Mittelman ${ }^{1}$, Jan Geliebter ${ }^{1}$ and Raj K. Tiwari ${ }^{1}$ \\ ${ }^{1}$ Department of Microbiology and Immunology, New York Medical College, Valhalla, NY, USA \\ Correspondence to: Raj K. Tiwari, email:Raj_Tiwari@nymc.edu \\ Keywords: epithelial-mesenchymal transition; inflammation; M1 macrophages; metastasis; secretory factors \\ Received: June 03, 2017 Accepted: September 05, 2017 Epub: March 23, 2018 Published: May 11, 2018
}

Copyright: Bednarczyk et al. This is an open-access article distributed under the terms of the Creative Commons Attribution License 3.0 (CC BY 3.0), which permits unrestricted use, distribution, and reproduction in any medium, provided the original author and source are credited.

\section{ABSTRACT}

The majority of breast cancers (90-95\%) arise due to mediators distinct from inherited genetic mutations. One major mediator of breast cancer involves chronic inflammation. M1 macrophages are an integral component of chronic inflammation and the breast cancer tumor microenvironment (TME). Previous studies have demonstrated that up to $\mathbf{5 0 \%}$ of the breast tumor comprise of tumor-associated macrophages (TAMs) and increased TAM infiltration has been associated with poor patient prognosis. Furthermore, breast cancer associated deaths are predominantly attributed to invasive cancers and metastasis with epithelial-mesenchymal transition (EMT) being implicated. In this study, we investigated the effects of cellular crosstalk between TAMs and breast cancer using an in vitro model system. M1 polarized THP1 macrophage conditioned media (CM) was generated and used to evaluate cellular and functional changes of breast cancer lines T47D and MCF-7. We observed that T47D and MCF-7 exhibited a partial EMT phenotype in the presence of activated THP$1 \mathrm{CM}$. Additionally, MCF-7 displayed a significant increase in migratory and invasive properties. We conclude that M1 secretory factors can promote a partial EMT of epithelial-like breast cancer cells. The targeting of M1 macrophages or their secretory components may inhibit EMT and limit the invasive potential of breast cancer.

\section{INTRODUCTION}

Breast cancer is the most common malignancy in women within the United States affecting around 1 in 8 women [1-2] with an estimated number of breast cancer cases in 2018 to be 268,670 [1]. Breast cancer is also the second leading cause of cancer-related deaths in females with approximately 40,450 attributed deaths and is second only to lung cancer [1]. These high death rates can be primarily attributed to invasive breast cancers that metastasize and form secondary tumors [3]. Increased breast screenings, newer aggressive therapeutics, and a reduction in hormone replacement therapy has resulted in a decline in breast cancer deaths since early 2000; however, metastatic breast cancer is still a major challenge $[4,5]$.

Around $5-10 \%$ of total breast cancer occurrences are a result of inherited genetic mutations (e.g., BRCA1/2), which leaves an astounding $90-95 \%$ of breast cancers that develop due to the influence of outside mediators. One major mediator of breast cancer development and progression is inflammation. Inflammation is a natural biological process that occurs during times of infection or wound healing and is self-limiting which is hence called acute inflammation. During chronic inflammation this self-limiting process is deregulated and can contribute to a number of diseases including: Alzheimer's [6], cardiomyopathy [7], osteoarthritis [8], inflammatory bowel disease (IBD) [9], and numerous types of cancers including breast [10-12]. Specifically, chronic inflammation has been associated with cancer initiation and progression [13-15].

One significant constituent of chronic inflammation is macrophages which are also a main resident of the breast tumor microenvironment (TME). Macrophages that reside within the TME are termed tumor-associated macrophages (TAMs) and have been shown to comprise up to $50 \%$ of the total tumor mass in certain breast cancer clinical cases 
with poor prognosis associated with increased TAM tumor infiltration [16-18].

Macrophages are subdivided into two distinct phenotypes, M1 or M2, depending on specific stimuli including cytokines and bacterial moieties. M1 macrophages are classically activated and proinflammatory and have traditionally been deemed as antitumor due to their ability to generate reactive nitrogen and oxygen intermediates, and pro-inflammatory cytokines $[19,20]$. On the contrary, M2 macrophages are alternatively activated and anti-inflammatory, promoting tumor progression by downregulating the immune response and stimulating angiogenesis [21, 22]. Although M1 macrophages are known to have antitumor properties, these immune cells enhance tumor development and progression through frequent tissue damage and disruption in addition to pro-inflammatory cytokines secreted.
Epithelial-mesenchymal transition (EMT) is a cellular process where epithelial cells lose adhesive properties and cell polarity to become motile and mesenchymal-like. Although EMT is known to be a natural occurrence serving important roles in development [23] and wound healing [24], cancer cells can harness this process in ultimately enhancing metastasis. Currently, the roles of M1 macrophages influencing breast cancer cells to undergo EMT is not widely known or studied.

Our laboratory has previously demonstrated that estrogen treated murine mammary breast cancer, and human thyroid cancer cells, secrete factors that promote human umbilical vein endothelial cell (HUVEC) migration and tube formation. These factors are contained within the conditioned media generated from these cancer cells. $[25,26]$. In this study, we used a similar methodology of generating conditioned media and investigated the role of M1 macrophage secretory factors and their potential
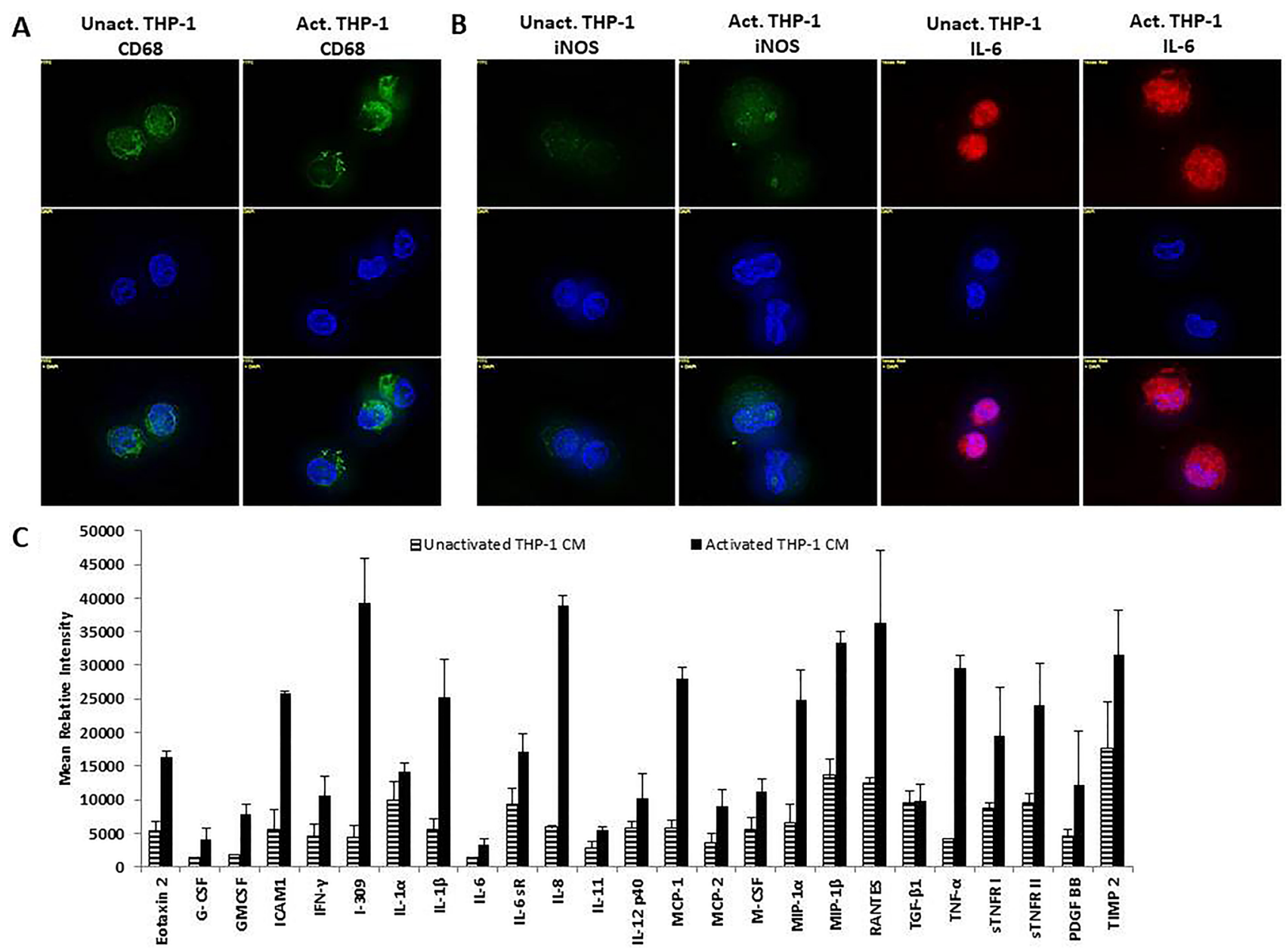

gUnactivated THP-1 CM

Activated THP-1 CM

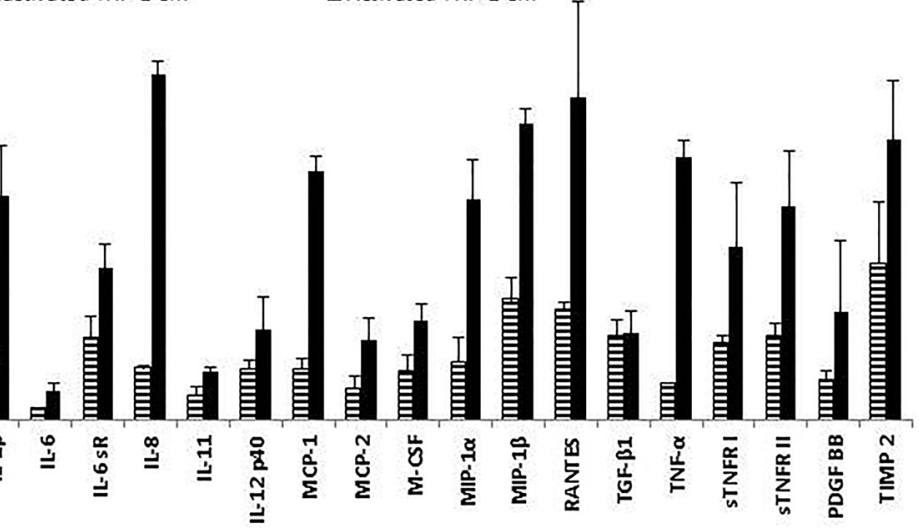

Figure 1: THP-1 cells exhibit an M1 macrophage phenotype following activation with TPA. (A) THP-1 cells express monocyte/macrophage lineage marker CD68. (B) Upon activation with TPA, THP-1 cells express M1 macrophage markers iNOS and IL6. Unactivated and activated THP-1 cells were seeded overnight and probed with CD68, iNOS, or IL-6 primary antibodies. Fluorescent secondary antibodies were used for immunofluorescence. Pictures were taken using Axiovision Rel 4.8 at 100x magnification. (C) Activated THP-1 cells express pro-inflammatory cytokines including: TNF- $\alpha$, IL-1 $\beta$, and IL-8. A human inflammation array was performed on unactivated or activated THP-1 CM (48 h). Cytokine/chemokine blots were analyzed using ImageJ and optical density determined and normalized \pm SEM of two independent experiments. 
in inducing EMT in epithelial-like breast cancer cell lines, T47D and MCF-7. We determined that a human monocytic cell line, THP-1, when polarized towards an M1 phenotype can induce breast cancer cells to undergo a partial EMT; thereby, having the potential to stimulate breast cancer metastasis.

\section{RESULTS}

\section{Activation of THP-1 with TPA induces an M1 macrophage phenotype}

In order to determine the effects of cellular crosstalk between TAMs and breast cancer mediated by macrophage secretory factors, the macrophage phenotype of THP-1 monocytes activated with 12-O-Tetradecanoylphorbel13-acetate (TPA) was first identified. Unactivated and activated THP-1 were probed for CD68, a transmembrane protein which is a common monocyte/macrophage lineage marker. Both, unactivated and activated, THP-1 cells expressed CD68 validating this cell type being of monocyte/macrophage lineage (Figure 1A). M1 macrophage markers iNOS and IL-6 were also evaluated using immunofluorescence analysis. Activated THP-1 cells expressed increased iNOS and IL-6 compared to unactivated THP-1 cells (Figure 1B). Additionally, an analysis of the cytokine profile of activated THP-1 cells showed a pro-inflammatory cytokine expression of TNF- $\alpha$, IL-1 $\beta$, and IL-8 (Figure 1C). This indicates that THP-1 cells, upon activation with TPA, promotes an M1

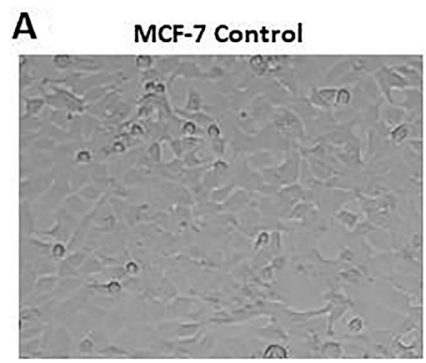

T47D Control

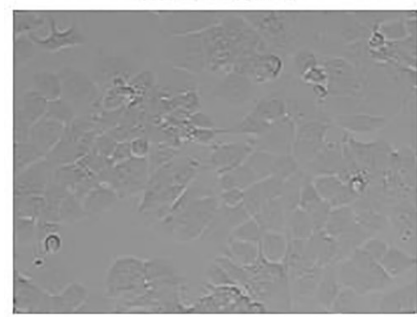

C

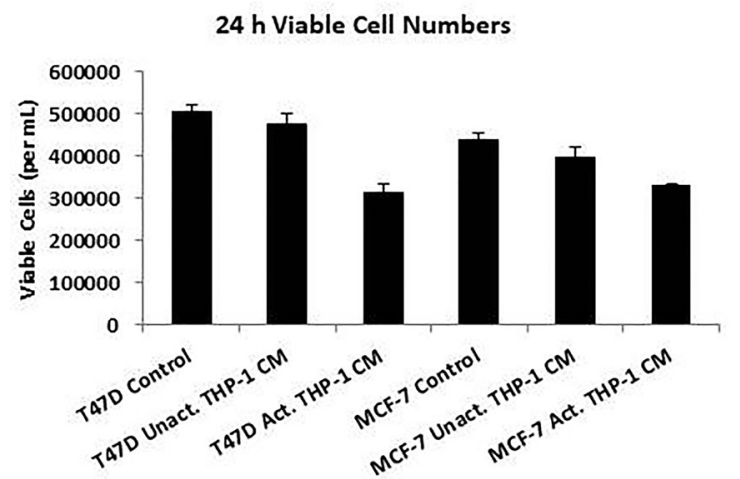

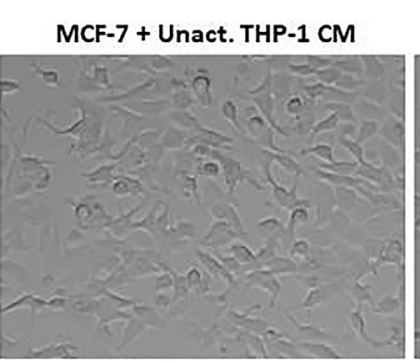

T47D + Unact. THP-1 CM
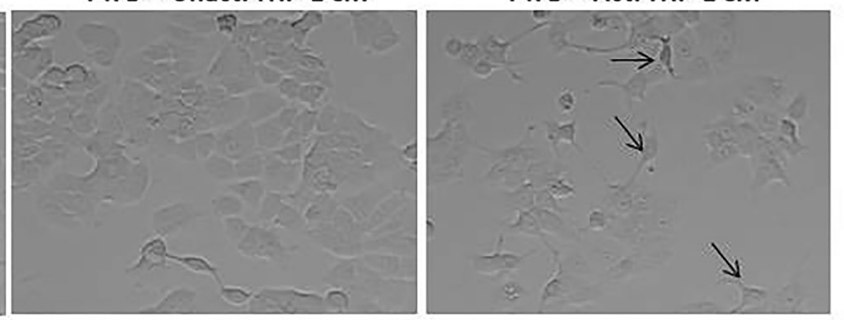

T47D + Act. THP-1 CM
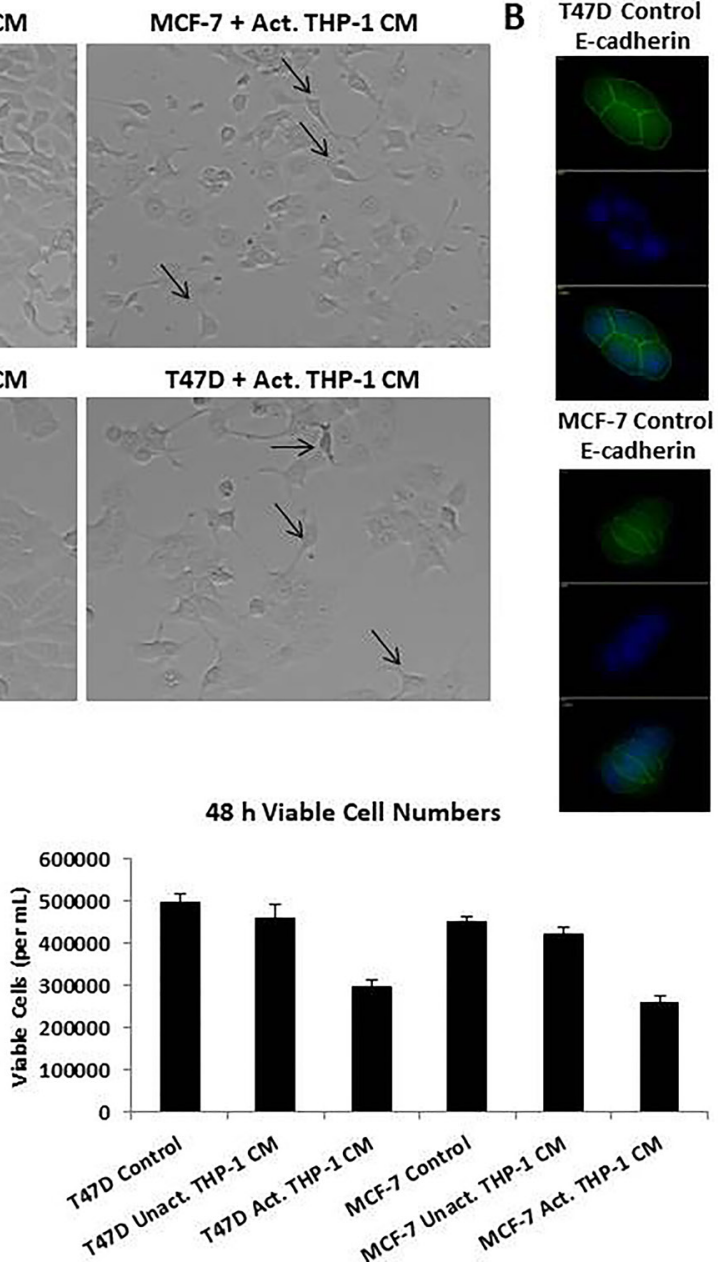

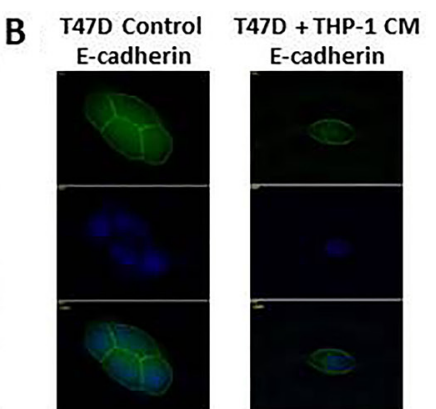

MCF-7 Control MCF-7 + THP-1 CM E-cadherin E-cadherin

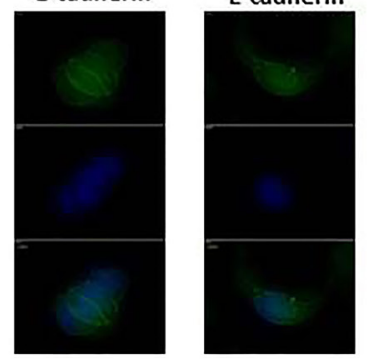

Figure 2: T47D and MCF-7 cells exhibited spindle-like morphology, decreased E-cadherin, and decreased viability viable cells following treatment with activated THP-1 CM. (A) Treatment of T47D and MCF-7 with activated THP-1 CM promotes mesenchymal-like morphological changes. Breast cancer cells were treated with unactivated or activated THP-1 CM for $24 \mathrm{~h}$ and cell morphology was observed by phase contrast microscopy. Pictures were taken at 20x magnification using phase contrast microscopy. (B) T47D and MCF-7 were treated for $24 \mathrm{~h}$ with control (5\% CS-RPMI) or activated THP-1 CM and probed with E-cadherin primary antibodies. Fluorescent secondary antibodies in addition to DAPI (nuclear stain) were used for immunofluorescence. Pictures were taken using Axiovision Rel 4.8 at 100x magnification. (C) Activated THP-1 CM leads to decreased number of viable T47D and MCF-7 cells at 24 and $48 \mathrm{~h}$. A trypan blue exclusion assay was performed to evaluate the number of viable cells. Data represents the number of viable cells in duplicates \pm SEM of two independent experiments. 
macrophage phenotype which is a major contributor to chronic inflammation.

\section{Activated THP-1 secretory factors alter breast cancer cell morphology and reduces viability}

In order to evaluate the effects of activated THP-1 secretory factors on breast cancer phenotype, we used an in vitro model system; T47D and MCF-7 human breast cancer cells treated with unactivated or activated THP-1 conditioned media (CM). T47D and MCF-7 human breast cancer cells are of the luminal subtype being estrogen receptor positive and Her2 negative and are presumably amenable to epithelial-mesenchymal transition. These cell lines in their native state in cell culture are epithelial-like. Activated THP-1 CM induced spindle-like morphology of breast cancer cells compared to unactivated THP-1 $\mathrm{CM}$ or control treatments (Figure 2A). T47D and MCF7 are epithelial-like cells and a transition to spindle-like morphology, indicates a change in cellular phenotype in breast cancer cells, resembling mesenchymal cells. There was decreased E-cadherin expression in T47D and MCF7 following activated THP-1 CM treatment (Figure 2B). A trypan blue exclusion assay was used to determine the number of viable breast cancer cells following THP$1 \mathrm{CM}$ treatments. We observed a significant decrease in the number of viable T47D and MCF-7 following $24 \mathrm{~h}$ treatment with activated THP-1 CM (Figure 2C).
In order to evaluate cellular interactions between M1 macrophages and breast cancer cells, we performed co-culture experiments with activated THP-1 and breast cancer cell lines using $0.4-\mu \mathrm{M}$ transwell chambers. We evaluated cell morphology and the viability. As observed with activated THP-1 CM treatments, T47D and MCF7 exhibited spindle-like morphology (Figure 3A) and a significant decrease in the number of viable cells (Figure 3B) in the presence of activated THP-1 cells. Thus, activated THP-1 secretory factors are promoting a mesenchymal-like phenotype in addition to decreased cellular growth of breast cancer cells.

\section{M1 macrophage inflammatory factors upregulate epithelial-mesenchymal transition related transcription factors}

Spindle-like morphology and reduced viability are prominent characteristics of cells undergoing EMT. Therefore, we evaluated whether activated THP-1 secretory factors are promoting the EMT process. We assessed EMT transcription factors and markers in T47D and MCF-7 treated with activated THP-1 CM using Western blot analysis and immunofluorescence. We observed increased nuclear localization of EMT transcription factors NF-kB and Snail in T47D (Figure 4A), while MCF-7 displayed increased nuclear localization of NF-kB, Snail, and Slug (Figure 4B). MDA-MB 231

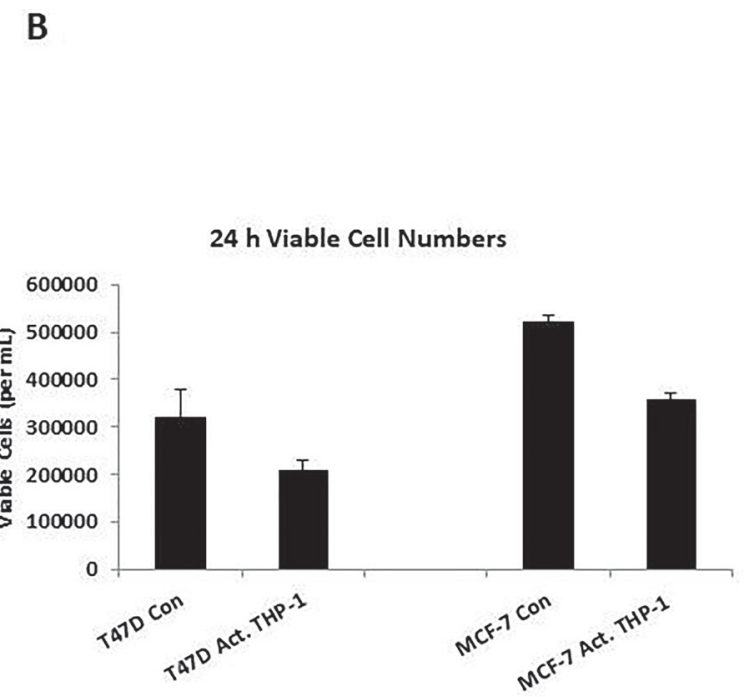

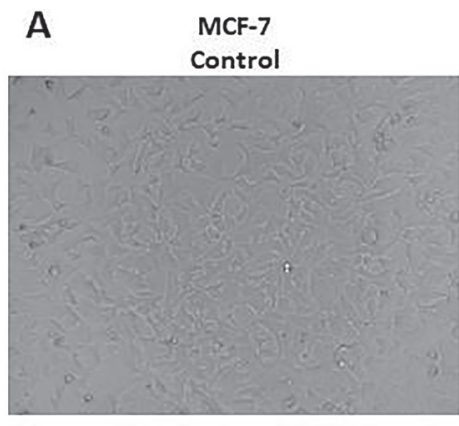
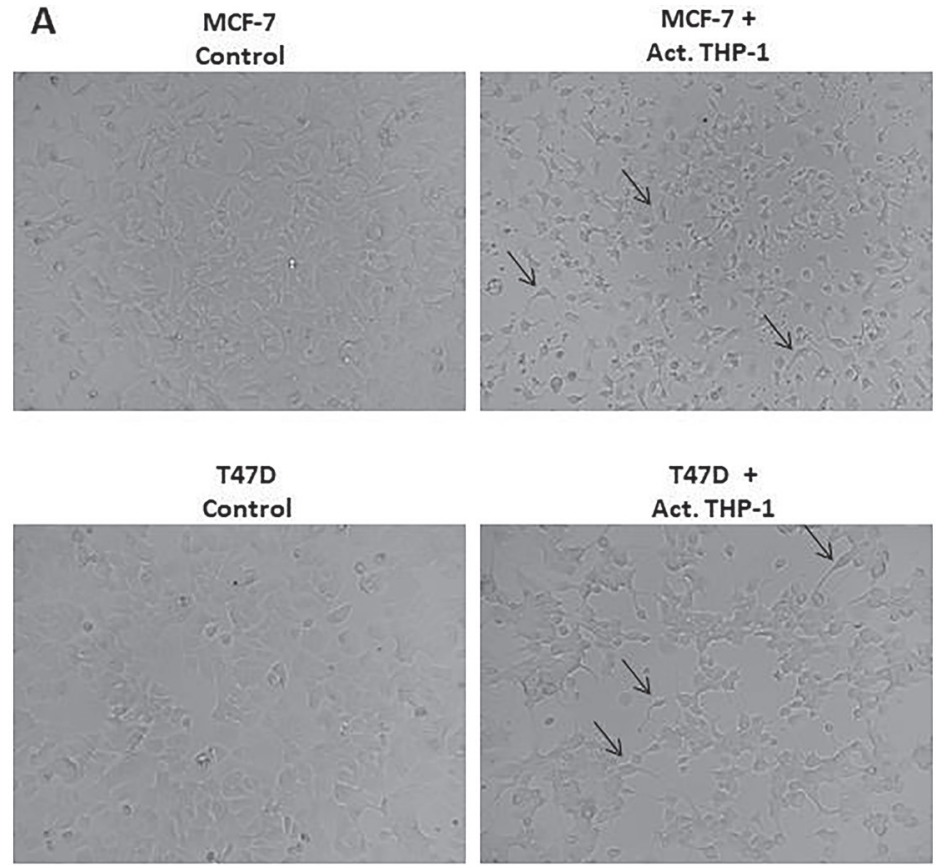

Figure 3: Spindle-like morphology and reduced viability is observed following co-culture with activated THP-1 cells. (A) Co-culture of activated THP-1 cells with either T47D or MCF-7 promotes mesenchymal-like morphology. Breast cancer cells were co-cultured for $24 \mathrm{~h}$ and cell morphology was observed. Pictures were taken at 10x magnification using phase contrast microscopy. (B) A decreased number of viable breast cancer cells was observed following co-culture with activated THP-1 cells for 24 h. A trypan blue exclusion assay was performed to evaluate viability. Data represents the number of viable cells in duplicates \pm SEM of two independent experiments in duplicates. 
that is considered post-EMT was used for comparison and displays robust mesenchymal Vimentin and Slug expression throughout (Figure 4C). Therefore, M1 macrophage secretory factors induce EMT transcription factor and marker changes.

\section{M1 macrophage inflammatory factors promote MCF-7 migration and invasion in addition to increased MMP-9 expression}

Since there was increased nuclear localization of EMT transcription factors and decreased E-cadherin expression on breast cancer cells, induction of EMT was indicative. We evaluated migration and invasion of T47D and MCF-7 following activated THP-1 CM treatments since these processes are known to be upregulated by cells undergoing EMT. Breast cancer cell migration and invasion was evaluated using a Boyden chamber assay. Following an
$18 \mathrm{~h}$ treatment with activated THP-1 CM, MCF-7 exhibited a significant increase in migration and invasion compared to untreated controls (Figure 5A, Figure 6A). Additionally, a scratch wound assay confirmed an increase in MCF-7 migratory capability in the presence of activated THP-1 CM (Figure 5B). T47D did not express significant changes in either migration or invasion following similar treatments (Figure 5A, Figure 6A).

Cells that have increased migratory and invasive capabilities are known to secrete matrix metalloproteinases (MMPs) such as MMP-2 and MMP-9 which breakdown the extracellular matrix allowing cells to migrate and invade tissues $[27,28]$. We thus evaluated MMP-9 expression in breast cancer cells following activated THP-1 CM treatments via Western blot analysis. We observed increased MMP9 expression in MCF-7 that correlated with the increased migration and invasion as previously shown (Figure 6B). Additionally, since there were no observed changes in

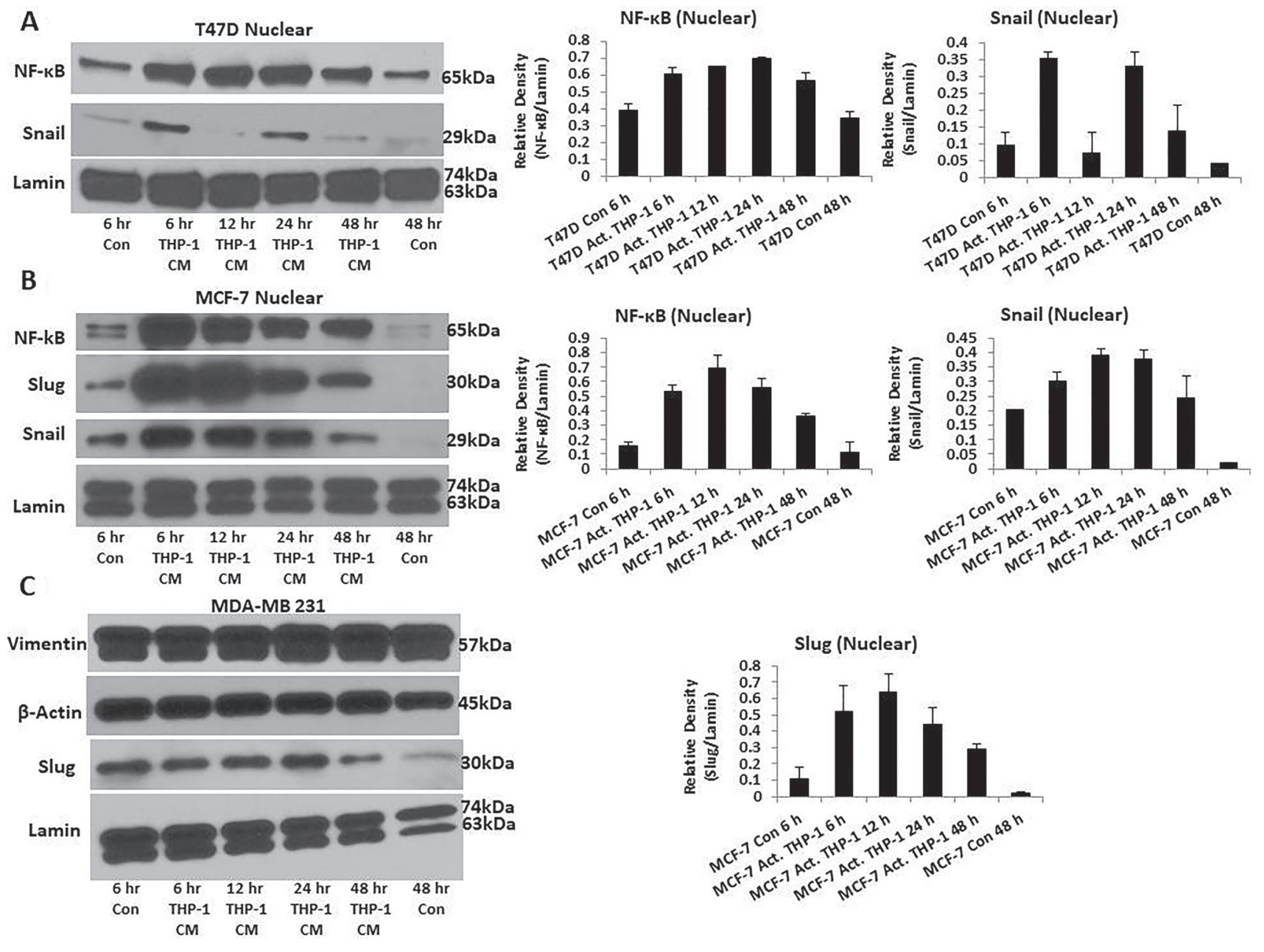

Figure 4: T47D and MCF-7 cells exhibit increased nuclear localization of EMT transcription factors. (A) Increased

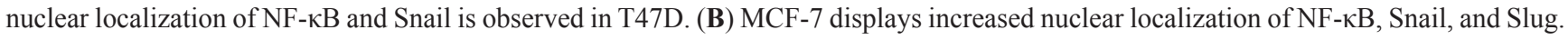
(C) MDA-MB 231 robustly expresses mesenchymal markers Vimentin and Slug. T47D, MCF-7, and MDA-MB 231 were treated with activated THP-1 CM for 6 h, 12 h, 24 h, and 48 h. Nuclear extractions were prepared and Western blots performed probing for: NF- $\kappa B$ (65 $\mathrm{kDa})$, Slug (30 kDa), Snail (29 kDa), Vimentin (57 kDa), $\beta$-Actin (45 kDa), and Lamin (63, $74 \mathrm{kDa})$. Relative density of NF- $\mathrm{BB}$, Snail, and Slug is shown normalized to Lamin loading controls \pm SEM of two independent experiments. 
migration or invasion for T47D with M1 secretory factors, we did not detect changes in MMP-9 (Figure 6B). Therefore, increased MMP-9 expression may contribute to the migratory and invasive potential of MCF-7.

\section{DISCUSSION}

Chronic inflammation is known to contribute to several types of diseases including cancer. One integral component of chronic inflammation involves immune cells, specifically macrophages. Macrophages are divided into M1 or M2 phenotypes and both types have been implicated in cancer development or progression. Anti-inflammatory cytokines such as IL-4, IL-13, and TGF- $\beta$ may polarize macrophages into M2 phenotypes which are considered immunosuppressive and can contribute to breast cancer progression by promoting growth, metastasis, and angiogenesis. On the contrary,
IFN- $\gamma$, GM-CSF, and LPS can polarize macrophages into M1 phenotype. Since the predominant role of M1 macrophages is related to inflammation and that chronic inflammation has a detrimental role in cancer progression, this study was focused on M1 macrophage based cellular transition. M1 macrophages are pro-inflammatory and have been traditionally deemed as having anti-tumor activity. However, M1 macrophages through chronic inflammation have been associated with cancer initiation due to introducing tissue damage $[19,20,29]$ and cytokine secretion $[19,30,31]$. In addition, the TME of numerous types of cancers including breast contain TAMs. Therefore, in this study we investigated the influence of M1 macrophages on breast cancer cells through cellular crosstalk mediated by macrophage secretory factors. We determined that cellular crosstalk mediated by M1 secretory factors promoted EMT of luminal subtypes of breast cancer.

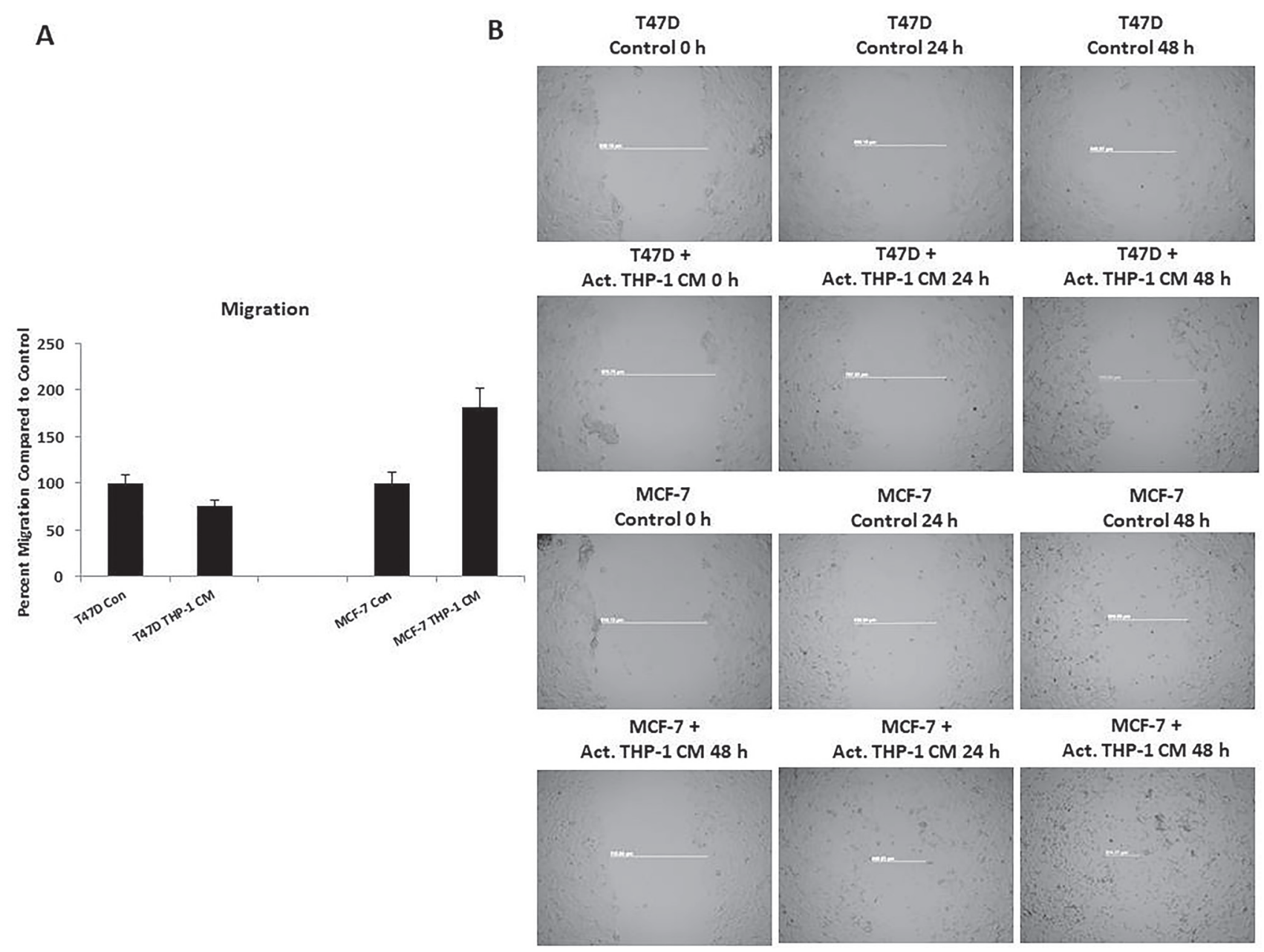

Figure 5: THP-1 CM promotes MCF-7 migration. (A) MCF-7 exhibits increased migration at $18 \mathrm{~h}$ in the presence of activated THP-1 CM. Breast cancer migration was evaluated using Boyden chamber assays. Pictures were taken using brightfield microscopy at $5 \mathrm{x}$ magnification counting four fields per insert. Data is represented as percent migration compared to control in duplicates \pm SEM of two independent experiments. (B) Scratch wound assay confirms increased MCF-7 migration. Scratch-wound assay was performed on T47D and MCF-7 treated with control (5\% CS-RPMI) or activated THP-1 CM with mitomycin C ( $2 \mu \mathrm{g} / \mathrm{mL})$ for $24 \mathrm{~h}$. Pictures were taken at $0 \mathrm{~h}$, $24 \mathrm{~h}$, and $48 \mathrm{~h}$ following control or CM treatments using phase contrast microscopy and Axiovision Rel 4.8 at 5x magnification. 
We used an in vitro model system to evaluate the interactions between infiltrating macrophages and breast cancer as would resemble within the TME. Previous studies have demonstrated that human monocytic cell lines such as THP-1 when activated with TPA exhibit an M1 phenotype [32-34]. We generated CM and observed that following the activation of THP-1 with TPA, there was enhanced expression of M1 marker iNOS in addition to pro-inflammatory cytokines IL-6, TNF- $\alpha$, IL- $1 \beta$, and IL-8 when compared to unactivated THP-1.

To evaluate cellular crosstalk mediated by proinflammatory macrophage secretory factors on breast cancer cells, we examined the cellular and functional changes caused by activated THP-1 CM on luminal/ epithelial-like breast cancer cell lines T47D and MCF7. We observed that activated THP-1 secretory factors induced EMT-like features in breast cancer cells. T47D and MCF-7 exhibited mesenchymal-like morphology and decreased viability following activated THP-1 CM and co-culture experiments. In addition, there was increased nuclear localization of EMT-related transcription factors for T47D and MCF-7. EMT-related transcription factors have functional roles in downregulating epithelial markers such as E-cadherin which is a component of adherens junctions and disruption of this protein promotes increased migratory capability of cells [35, 36]. We evaluated E-cadherin expression in breast cancer cells following activated THP-1 CM treatment. There was a loss of E-cadherin in spindle-like T47D and MCF-7 cells following treatments. Decreased E-cadherin expression would subsequently influence breast cancer cell motility warranting an inquiry into cell migration and invasion.

Cell migration and invasion are processes that are identified as being upregulated in cells undergoing EMT. We observed that activated THP-1 CM promoted MCF-

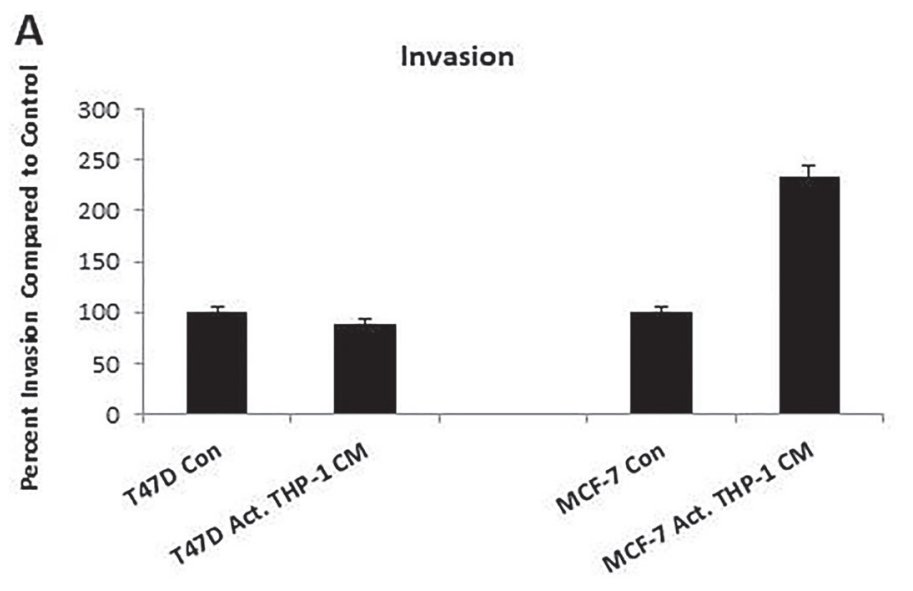

7 to demonstrate significantly increased migration as observed through Boyden chamber and scratch wound assays. Additionally, there was increased invasion and MMP-9 expression by MCF-7. T47D did not display changes in migration or invasion in the presence of activated THP-1 CM compared to control. As was previously shown, T47D displayed an increase in Snail nuclear localization which was observed to be oscillatory through $48 \mathrm{~h}$. The irregular nuclear localization of Snail and the absence of Slug may have negatively affected T47D migratory and invasive properties as opposed to MCF-7 in which Snail and Slug expression was dynamic. Therefore, MCF-7 displayed a more progressed EMT compared to T47D.

Several studies have formerly established a link between inflammatory factors and cancer EMT [37-40]. Pro-inflammatory cytokines such as TNF- $\alpha$ have been previously demonstrated to induce EMT in a number of cancers including breast cancer $[41,42]$. TNF- $\alpha$ has been shown to stimulate the induction of EMT transcription factors including Snail, Slug, Twist, and ZEB1/2 through $\mathrm{NF}-\kappa \mathrm{B}$ signaling $[41,43]$. It is possible that TNF- $\alpha$ which is secreted by activated THP-1 promotes the induction of EMT as observed in T47D and MCF-7. Further studies examining the neutralization of TNF- $\alpha$ in activated THP-1 $\mathrm{CM}$ and the effects on EMT induction should be carried out.

Other cytokines that have been implicated in EMT and were present in activated THP-1 CM include: IL-1 $\beta$ [44], IL-6 [45], IL-8 [46] and TGF- $\beta$ [47]. Additionally, the influence of macrophage secreted exosomes containing miRNA cargo was not evaluated. Exosomes are small (30-120 nm) membraneous nanovesicles that are secreted from cells and have been implicated in cell-cell communication [48]. Depending on the cell type, exosomes can contain various types of cargo including miRNA. Several studies have reported that miRNA

B

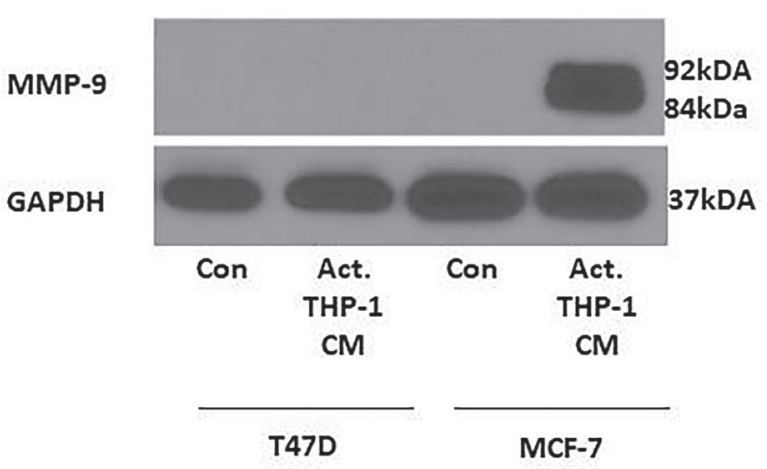

Figure 6: Activated THP-1 CM treatment enhances MCF-7 invasion and MMP-9 expression. (A) MCF-7 exhibits increased invasion at $18 \mathrm{~h}$ in the presence of activated THP-1 CM. Breast cancer invasion was evaluated using Boyden chamber assays. Pictures were taken using brightfield microscopy at $5 \mathrm{x}$ magnification counting four fields per insert. Data is represented as percent invasion compared to control in duplicates \pm SEM of two independent experiments. (B) There is increased expression of MMP-9 by MCF-7 with $24 \mathrm{~h}$ activated THP-1 CM treatment. MMP-9 (84, $92 \mathrm{kDa})$ and GAPDH (37 kDa) expression was evaluated using Western blots. 
can contribute to EMT $[49,50]$. Therefore, it is possible that macrophage secreted exosomes containing particular miRNA may promote EMT as observed with T47D and MCF-7.

We demonstrate that pro-inflammatory secretory factors can induce a partial EMT in breast cancer cells, displaying a combination of epithelial and mesenchymal characteristics. Additionally, certain breast cancer cell lines may be more prone to undergoing EMT compared to others. In our study, we observed that MCF-7 exhibited increased EMT properties compared to T47D following similar treatments. Breast cancer cells that undergo EMT have a greater likelihood of contributing to metastasis, decreasing survival outcomes for patients. Additionally, breast cancer cells that have been shown to undergo EMT may not only contribute to increased metastasis, but may become resistant to cytotoxic T lymphocytes [51]. Therefore, the degree of tumor cell EMT can potentially affect how a patient may or may not respond to immunotherapy which has an important implication in patient treatment. Further it should be noted that epithelial-mesenchymal transition has inherent plasticity which is well integrated with stem cell population generation, [52] and has considerable redundancies as all factors, mediators and signal transducers are not induced concurrently.

We propose that by directly targeting M1 macrophages or neutralizing pro-inflammatory secretory factors, the chronic inflammatory response may be limited and potentially reduced ultimately hindering breast cancer progression. By reducing breast cancer invasive potential through the inhibition of EMT processes, the risk of metastasis decreases and the prospect of patient survival increases. A better understanding of the cells found within the TME and how they impact breast cancer progression will help in terms of breast cancer therapy.

\section{MATERIALS AND METHODS}

\section{Cells and cell culture}

The breast cancer cell lines used include: T47D and MCF-7 which were obtained from American Type Culture Collection (ATCC). T47D and MCF-7 cells were grown in RPMI-1640 (Mediatech, Herndon, VA) and DMEM-1640, respectively, supplemented with 10\% fetal bovine serum (FBS) (Atlanta Biologicals, Atlanta, GA), penicillin 10,000 IU/mL, streptomycin 10,000 $\mu \mathrm{g} /$ $\mathrm{mL}$ (Mediatech) and $2 \mathrm{mM}$ L-glutamine (Mediatech). Human monocytic cell line, THP-1, was grown in RPMI-1640 (Mediatech, Herndon, VA), supplemented with 10\% FBS (Atlanta Biologicals, Atlanta, GA), penicillin 10,000 IU/mL, streptomycin 10,000 ug/mL (Mediatech), $2 \mathrm{mM}$ L-glutamine (Mediatech), and 0.05 mM 2-mercaptoethanol. T47D, MCF-7, and THP-1 cell lines were purchased between 2000 to 2013 and were authenticated by the Genomics Core at Albert Einstein School of Medicine using SoftGenetics GeneMarkerHID.

\section{Differentiation of THP-1 and generation of conditioned media}

THP-1 monocytes were seeded at a density of $5 \times 10^{6}$ cells and treated with $200 \mathrm{nM}$ 12-O-Tetradecanoylphorbel13-acetate (TPA) in RPMI-1640 supplemented media for THP-1 as specified above. The media was discarded and rinsed with phenol red free RPMI-1640 following incubation for $48 \mathrm{~h}$. THP-1 cells were further incubated for $48 \mathrm{~h}$ in 5\% charcoal-stripped RPMI to obtain THP1 macrophage conditioned media (CM). The conditioned media was then collected and centrifuged for $5 \mathrm{~min}$ at $1500 \mathrm{rpm}$ to remove cells and cell debris. Conditioned media was stored in $-80^{\circ} \mathrm{C}$ until future use.

\section{Morphology analysis}

T47D and MCF-7 were seeded in 6 well plates at $3 \times 10^{5}$ per well in duplicates and allowed to adhere and grow overnight in RPMI or DMEM media as previously described, respectively. Seeding media was removed from the cell monolayer and rinsed with phenol-red free RPMI. Breast cancer cells were treated with either 5\% charcoalstripped phenol-red free RPMI as control, unactivated THP-1 CM, or activated THP-1 CM for CM evaluation for $24 \mathrm{~h}$. Pictures were taken under phase contrast microscopy at $20 \times$ magnification.

\section{Trypan blue exclusion assay}

T47D and MCF-7 were seeded in 6 well plates at $3 \times$ $10^{5}$ per well in duplicates and allowed to adhere and grow overnight in RPMI or DMEM culture media as previously described, respectively. Seeding media was removed from the cell monolayer and rinsed with phenol-red free RPMI. Breast cancer cells were treated with either 5\% charcoalstripped phenol-red free RPMI, unactivated THP-1 CM, or activated THP-1 CM for 24 or 48 h. Following respective time points, cells were harvested and stained using $0.4 \%$ trypan blue (Sigma Chemical). Viable (unstained) cells were counted using a hemocytometer.

\section{Co-culture experiments}

THP-1 were activated using TPA for $48 \mathrm{~h}$ and seeded in 0.4- $\mu \mathrm{m}$ transwell 6-well plates (Corning ${ }^{\mathrm{TM}}$ Costar $^{\mathrm{TM}}$ ) at $1 \times 10^{6}$ cells per well in duplicates. T47D and MCF-7 were seeded in 6 well plates at $3 \times 10^{5}$ cells per well in duplicates and allowed to adhere and grow overnight in RPMI or DMEM culture media as previously described. Seeding media was removed from the cell monolayer and rinsed with phenol-red free RPMI. Activated THP-1 were rinsed with phenol-red free RPMI. Breast cancer cells were incubated with 5\% charcoal-stripped phenol-red free RPMI. 0.4- $\mu \mathrm{m}$ transwells for controls and activated THP1 contained 5\% charcoal-stripped phenol-red free RPMI 
and respective co-cultures transpired for $24 \mathrm{~h}$. Morphology pictures were taken using phase contrast microscopy at $10 \times$ magnification. Viable cells were determined using trypan blue exclusion assay as mentioned above.

\section{Western blot analysis of total cell or subcellular fractionated lysates}

The generation of total cell lysates or subcellular fractionated lysates was prepared by two different methods.

\section{Total cell lysates}

Following activated THP-1 CM treatments, breast cancer cells were harvested and lysates generated using RIPA Lysis Buffer (50 mM Tris-HCL pH 7.5, $150 \mathrm{mM}$ $\mathrm{NaCl}, 1 \% \mathrm{NP}-40,0.5 \%$ Sodium deoxycholate, $0.1 \% \mathrm{SBS}$ ) with Halt ${ }^{\mathrm{TM}}$ Protease and Phosphatase Inhibitor Cocktail (1X Concentration) (Life Technologies). Membranes were blocked with $5 \%$ milk in TBST $(10 \mathrm{mM}$ Tris-HCL, pH $7.5,200 \mathrm{mM} \mathrm{NaCl}, 0.05 \%$ Tween-20) for $2 \mathrm{~h}$ at room temperature and then incubated overnight in primary antibodies: MMP-9 (Cell Signaling) and GAPDH (Cell Signaling). Membranes were washed 3 times for $10 \mathrm{~min}$ per wash and incubated with secondary antibodies of goat anti-rabbit IgG $(\mathrm{H}+\mathrm{L})$ Horseradish Peroxidase (Thermo Scientific) for $2 \mathrm{~h}$ at room temperature.

\section{Subcellular fractionated lysates}

Following activated THP-1 CM treatments, breast cancer cells were harvested and cytoplasmic and nuclear protein extracts generated using NE-PER Nuclear and Cytoplasmic Extraction Reagents (Thermo Scientific) as per manufacturer's instruction with Halt ${ }^{\mathrm{TM}}$ Protease and Phosphatase Inhibitor Cocktail (1X Concentration) (Life Technologies). Membranes were blocked with 5\% milk in TBST (10 mM Tris-HCL, pH 7.5, $200 \mathrm{mM}$ $\mathrm{NaCl}, 0.05 \%$ Tween-20) for $2 \mathrm{~h}$ at room temperature and then incubated overnight in primary antibodies of either: NF- $\kappa B$ (Cell Signaling), Snail (Cell Signaling), Slug (Cell Signaling), and Lamin A/C (Cell Signaling) at $4^{\circ} \mathrm{C}$. Membranes were washed 3 times for 10 min per wash and incubated with secondary antibodies of either goat anti-rabbit $\operatorname{IgG}(\mathrm{H}+\mathrm{L})$ Horseradish Peroxidase (Thermo Scientific) or rabbit anti-mouse $\operatorname{IgG}(\mathrm{H}+\mathrm{L})$ Horseradish Peroxidase (Thermo Scientific) for $2 \mathrm{~h}$ at room temperature.

\section{Migration and invasion assays}

BD Biocoat Control Inserts (BD Biosciences) and $\mathrm{BD}$ Biocoat Matrigel Invasion Chambers (BD Biosciences) with $8-\mu \mathrm{m}$ pore membrane filters were used for the migration and invasion assays, respectively.
Matrigel inserts were rehydrated for $2 \mathrm{~h}, 37^{\circ} \mathrm{C}$ with phenol-red free RPMI. Inserts were then incubated with $2.5 \times 10^{4}$ cells $/ 0.5 \mathrm{~mL}$ of T47D or MCF-7 in the presence of 5\% charcoal-stripped phenol-red free RPMI (control) or $48 \mathrm{~h}$ activated THP-1 CM with 10\% charcoal-stripped RPMI in the chamber as the chemoattractant. Following $18 \mathrm{~h}$ treatments at $37^{\circ} \mathrm{C}, 5 \% \mathrm{CO}_{2}$ atmosphere, the removal of non-migrating/invading cells on the upper surface was performed through scraping with a cotton swab. Cells were fixed with $100 \%$ methanol for $2 \mathrm{~min}$ and stained with $1 \%$ Toluidine Blue in 1\% Borex for 2 min. Excess stain was removed through rinsing the inserts in distilled water and allowed to dry overnight. Pictures were taken using PixeLINK Megapixel FlatWire Camera Release 3.2 on the Axiovert $200 \mathrm{M}$ microscope (Carl Zeiss MicroImaging, Inc., Thornwood, NY) at 5x magnification counting four fields per insert.

\section{Scratch-wound assay}

Migration was assessed for T47D and MCF-7 using a scratch-wound assay. Cells were seeded in 6 well plates at $5 \times 10^{5}$ cells per well in duplicates in RPMI or DMEM media as previously described. Cells were allowed to adhere and grow to $60-75 \%$ confluent cell monolayers. Two vertical wounds were created using a $10 \mu \mathrm{L}$ sterile pipette tip followed by removal of detached cells and cell debris by rinsing with phenol-red free RPMI. The wounded cell monolayer was treated with either 5\% charcoal-stripped phenol-red free RPMI or activated THP-1 CM with mitomycin C ( $2 \mu \mathrm{g} / \mathrm{mL})$ for $24 \mathrm{~h}$. Phase contrast microscopy captured pictures at $0 \mathrm{~h}$ and $24 \mathrm{~h}$ following treatments using Axiovision Rel 4.8 on the Axiovert $200 \mathrm{M}$ microscope (Carl Zeiss MicroImaging, Inc., Thornwood, NY) at $5 \times$ magnification.

\section{Abbreviations}

CM, conditioned media; EMT, epithelialmesenchymal transition; MMPs, matrix metalloproteinases; TPA, 12-O-Tetradecanoylphorbel13-acetate; TAMs, tumor-associated macrophages; TME, tumor microenvironment.

\section{Author contributions}

R.B.B. conceived the ideas and experiments with R.J.K. R.B.B. conducted the experiments, acquired data, and performed data analysis. R.B.B. wrote the manuscript. N. Y. T., E. K. H., G. B. R., R. M., A.M., J. G., and R. K. $\mathrm{T}$. reviewed and edited the manuscript.

\section{CONFLICTS OF INTEREST}

No potential conflicts of interest were disclosed. 


\section{FUNDING}

Support from the National Cancer Institute (Grant 5R01CA131946-02 to Raj K. Tiwari and Jan Geliebter) is recognized.

\section{REFERENCES}

1. Siegel RL, Miller KD, Jemal A. Cancer statistics, 2018. CA Cancer J Clin. 2016; 68:7-30.

2. DeSantis CE, Fedewa SA, Goding Sauer A, Kramer JL, Smith RA, Jemal A. Breast cancer statistics, 2015: Convergence of incidence rates between black and white women. CA Cancer J Clin. 2016; 66:31-42.

3. Weigelt B, Peterse JL, van 't Veer LJ. Breast cancer metastasis: Markers and models. Nat Rev Cancer. 2005; 5:591-602.

4. Dossus L, Benusiglio PR. Lobular breast cancer: Incidence and genetic and non-genetic risk factors. Breast Cancer Res. $2015 ; 17: 37$.

5. Krieger N, Chen JT, Waterman PD. Decline in US breast cancer rates after the women's health initiative: Socioeconomic and racial/ethnic differentials. Am J Public Health. 2010; 100:972.

6. Heppner FL, Ransohoff RM, Becher B. Immune attack: The role of inflammation in alzheimer disease. Nat Rev Neurosci. 2015; 16:358-372.

7. Bironaite D, Daunoravicius D, Bogomolovas J, Cibiras S, Vitkus D, Zurauskas E, Zasytyte I, Rucinskas K, Labeit S, Venalis A, Grabauskiene V. Molecular mechanisms behind progressing chronic inflammatory dilated cardiomyopathy. BMC Cardiovasc Disord. 2015; 15:26.

8. Sokolove J, Lepus CM. Role of inflammation in the pathogenesis of osteoarthritis: Latest findings and interpretations. Ther Adv Musculoskelet Dis. 2013; 5:77-94.

9. Rubin DC, Shaker A, Levin MS. Chronic intestinal inflammation: Inflammatory bowel disease and colitisassociated colon cancer. Front Immunol. 2012; 3:107.

10. Terzic J, Grivennikov S, Karin E, Karin M. Inflammation and colon cancer. Gastroenterology. 2010; 138:2101-2114.

11. DeNardo DG, Coussens LM. Inflammation and breast cancer. Balancing immune response: crosstalk between adaptive and innate immune cells during breast cancer progression. Breast Cancer Res. 2007; 9:212.

12. Farrow B, Evers BM. Inflammation and the development of pancreatic cancer. Surg Oncol. 2002; 10:153-169.

13. Baumgarten SC, Frasor J. Minireview: Inflammation: An instigator of more aggressive estrogen receptor (ER) positive breast cancers. Mol Endocrinol. 2012; 26:360-371.

14. Lu H, Ouyang W, Huang C. Inflammation, a key event in cancer development. Mol Cancer Res. 2006; 4:221-233.

15. Coussens LM, Werb Z. Inflammation and cancer. Nature. 2002; 420:860-867.
16. Obeid E, Nanda R, Fu YX, Olopade OI. The role of tumorassociated macrophages in breast cancer progression (review). Int J Oncol. 2013; 43:5-12.

17. Bingle L, Brown NJ, Lewis CE. The role of tumourassociated macrophages in tumour progression: Implications for new anticancer therapies. J Pathol. 2002; 196:254-265.

18. Lewis CE, Leek R, Harris A, McGee JO. Cytokine regulation of angiogenesis in breast cancer: The role of tumor-associated macrophages. J Leukoc Biol. 1995; 57:747-751.

19. Biswas SK, Sica A, Lewis CE. Plasticity of macrophage function during tumor progression: Regulation by distinct molecular mechanisms. J Immunol. 2008; 180:2011-2017.

20. Balkwill F, Charles KA, Mantovani A. Smoldering and polarized inflammation in the initiation and promotion of malignant disease. Cancer Cell. 2005; 7:211-217.

21. Lamagna $C$, Aurrand-Lions M, Imhof BA. Dual role of macrophages in tumor growth and angiogenesis. J Leukoc Biol. 2006; 80:705-713.

22. Sica A, Schioppa T, Mantovani A, Allavena P. Tumourassociated macrophages are a distinct M2 polarised population promoting tumour progression: Potential targets of anti-cancer therapy. Eur J Cancer. 2006; 42:717-727.

23. Drasin DJ, Robin TP, Ford HL. Breast cancer epithelialto-mesenchymal transition: Examining the functional consequences of plasticity. Breast Cancer Res. 2011; 13:226.

24. Thiery JP, Acloque H, Huang RY, Nieto MA. Epithelialmesenchymal transitions in development and disease. Cell. 2009; 139:871-890.

25. George AL, Rajoria S, Suriano R, Mittleman A, Tiwari RK. Hypoxia and estrogen are functionally equivalent in breast cancer-endothelial cell interdependence. Mol Cancer. 2012; 11:80.

26. Rajoria S, Hanly E, Nicolini A, George AL, Geliebter J, Shin EJ, Suriano R, Carpi A, Tiwari RK. Interlinking of hypoxia and estrogen in thyroid cancer progression. Curr Med Chem. 2014; 21:1351-1360.

27. Gong Y, Chippada-Venkata UD, Oh WK. Roles of matrix metalloproteinases and their natural inhibitors in prostate cancer progression. Cancers (Basel). 2014; 6:1298-1327.

28. Shah FD, Shukla SN, Shah PM, Shukla HK, Patel PS. Clinical significance of matrix metalloproteinase 2 and 9 in breast cancer. Indian J Cancer. 2009; 46:194-202.

29. Williams CB, Yeh ES, Soloff AC. Tumor-associated macrophages: Unwitting accomplices in breast cancer malignancy. NPJ Breast Cancer. 2016; 2:15025.

30. Liou GY, Storz P. Inflammatory macrophages in pancreatic acinar cell metaplasia and initiation of pancreatic cancer. Oncoscience. 2015; 2:247-251. https://doi. org/10.18632/oncoscience. 151 .

31. Karin M, Greten FR. NF-kappaB: Linking inflammation and immunity to cancer development and progression. Nat Rev Immunol. 2005; 5:749-759. 
32. Daigneault M, Preston JA, Marriott HM, Whyte MK, Dockrell DH. The identification of markers of macrophage differentiation in PMA-stimulated THP-1 cells and monocyte-derived macrophages. PLoS One. 2010; 5:e8668.

33. Schwende H, Fitzke E, Ambs P, Dieter P. Differences in the state of differentiation of THP-1 cells induced by phorbol ester and 1,25-dihydroxyvitamin D3. J Leukoc Biol. 1996; 59:555-561.

34. Engstrom A, Erlandsson A, Delbro D, Wijkander J. Conditioned media from macrophages of M1, but not M2 phenotype, inhibit the proliferation of the colon cancer cell lines HT-29 and CACO-2. Int J Oncol. 2014; 44:385-392.

35. Lamouille S, Xu J, Derynck R. Molecular mechanisms of epithelial-mesenchymal transition. Nat Rev Mol Cell Biol. 2014; 15:178-196.

36. Min C, Eddy SF, Sherr DH, Sonenshein GE. NF-kappaB and epithelial to mesenchymal transition of cancer. J Cell Biochem. 2008; 104:733-744.

37. Cohen EN, Gao H, Anfossi S, Mego M, Reddy NG, Debeb B, Giordano A, Tin S, Wu Q, Garza RJ, Cristofanilli M, Mani SA, Croix DA, et al. Inflammation mediated metastasis: Immune induced epithelial-to-mesenchymal transition in inflammatory breast cancer cells. PLoS One. 2015; 10:e132710.

38. Ricciardi M, Zanotto M, Malpeli G, Bassi G, Perbellini O, Chilosi M, Bifari F, Krampera M. Epithelial-to-mesenchymal transition (EMT) induced by inflammatory priming elicits mesenchymal stromal cell-like immune-modulatory properties in cancer cells. Br J Cancer. 2015; 112:1067-1075.

39. Wu Y, Deng J, Rychahou PG, Qiu S, Evers BM, Zhou BP. Stabilization of snail by NF-kappaB is required for inflammation-induced cell migration and invasion. Cancer Cell. 2009; 15:416-428.

40. Bates RC, Mercurio AM. Tumor necrosis factor-alpha stimulates the epithelial-to-mesenchymal transition of human colonic organoids. Mol Biol Cell. 2003; 14:1790-1800.

41. Li CW, Xia W, Huo L, Lim SO, Wu Y, Hsu JL, Chao CH, Yamaguchi H, Yang NK, Ding Q, Wang Y, Lai YJ, LaBaff AM, et al. Epithelial-mesenchymal transition induced by TNF-alpha requires NF-kappaB-mediated transcriptional upregulation of Twist1. Cancer Res. 2012; 72:1290-1300.

42. Wang H, Wang HS, Zhou BH, Li CL, Zhang F, Wang XF, Zhang G, Bu XZ, Chai SH, Du J. Epithelial-mesenchymal transition (EMT) induced by TNF-alpha requires AKT/ GSK-3beta-mediated stabilization of snail in colorectal cancer. PLoS One. 2013; 8:e56664.

43. Wu Y, Zhou BP. TNF-alpha/NF-kappaB/Snail pathway in cancer cell migration and invasion. Br J Cancer. 2010; 102:639-644.

44. Li Y, Wang L, Pappan L, Galliher-Beckley A, Shi J. IL1 beta promotes stemness and invasiveness of colon cancer cells through Zeb1 activation. Mol Cancer. 2012; 11:87.

45. Sullivan NJ, Sasser AK, Axel AE, Vesuna F, Raman V, Ramirez N, Oberyszyn TM, Hall BM. Interleukin-6 induces an epithelial-mesenchymal transition phenotype in human breast cancer cells. Oncogene. 2009; 28:2940-2947.

46. Fernando RI, Castillo MD, Litzinger M, Hamilton DH, Palena C. IL-8 signaling plays a critical role in the epithelial-mesenchymal transition of human carcinoma cells. Cancer Res. 2011; 71:5296-5306.

47. Pang MF, Georgoudaki AM, Lambut L, Johansson J, Tabor V, Hagikura K, Jin Y, Jansson M, Alexander JS, Nelson CM, Jakobsson L, Betsholtz C, Sund M, et al. TGF-beta1induced EMT promotes targeted migration of breast cancer cells through the lymphatic system by the activation of CCR7/CCL21-mediated chemotaxis. Oncogene. 2016; 35:748-760.

48. Bang C, Thum T. Exosomes: New players in cell-cell communication. Int J Biochem Cell Biol. 2012; 44:2060-2064.

49. De Craene B, Berx G. Regulatory networks defining EMT during cancer initiation and progression. Nat Rev Cancer. 2013; 13:97-110.

50. Slaby O, Svoboda M, Fabian P, Smerdova T, Knoflickova D, Bednarikova M, Nenutil R, Vyzula R. Altered expression of miR-21, miR-31, miR-143 and miR-145 is related to clinicopathologic features of colorectal cancer. Oncology. 2007; 72:397-402.

51. Terry S, Chouaib S. EMT in immuno-resistance. Oncoscience. 2015; 2:841-842. https://doi.org/10.18632/ oncoscience. 226 .

52. Lin SJ, Yang DR, Wang N, Jiang M, Miyamoto H, Li G, Chang C. TR4 nuclear receptor enhances prostate cancer initiation via altering the stem cell population and EMT signals in the PPARG-deleted prostate cells. Oncoscience. 2015; 2:142-50. https://doi.org/10.18632/oncoscience.121. 